\title{
Development of high-temperature oxide melt solution calorimetry for p-block element containing materials - CORRIGENDUM
}

Mykola Abramchuk, Kristina Lilova, Tamilarasan Subramani, Ray Yoo, Alexandira Navrostky Published online: 17 February 2021

doi: https://doi.org/10.1557/jmr.2020.185 Published online by

Cambridge University Press: 22 July 2020

The authors of this article [1] should appear as follows: Mykola Abramchuk, Kristina Lilova, Tamilarasan Subramani,

Ray Yoo and Alexandra Navrotsky

ORCID's have also been updated.

\section{Reference}

1. M. Abramchuk, K. Lilova, T. Subramani, R. Yoo, and

A. Navrostky: Development of high-temperature oxide melt solution calorimetry for p-block element containing materials. Journal of Materials Research, 1-8. Cambridge University Press (2020). 